\title{
Evaluation of fine needle aspiration biopsy in oral cavity and head and neck region with different stains techniques
}

\begin{abstract}
Ana Paula Candido dos SANTOS Norberto Nobuo SUGAYA Décio dos Santos PINTO Jr. Celso Augusto LEMOS Jr.
\end{abstract}

Universidade de São Paulo - USP, School of Dentistry, Department of Stomatology, São Paulo, SP, Brazil.

Declaration of Interests: The authors certify that they have no commercial or associative interest that represents a conflict of interest in connection with the manuscript.

Corresponding Author: Ana Paula Candido dos Santos

E-mail: anapaulacs@usp.br

DOI: 10.1590/1807-3107BOR-2015.vol29.0080

Submitted: Jul 07, 2014

Accepted for publication: Jan 29, 2015

Last revision: May 19, 2015

\begin{abstract}
The present study aimed to evaluate the Fine Needle Aspiration Biopsy in different staining techniques in nodular lesions of the oral cavity and head and neck region, as their sensitivity, specificity and accuracy, staining with Panoptic, Papanicolaou and Hematoxylin-Eosin (H\&E) stains. 46 patients who sought the Clinic of the Discipline of Clinical Stomatology at FOUSP were selected consecutively, with nodular lesions in the oral cavity and head and neck region. The material obtained by FNAB was sent on 6 different slides, stained by the method of Panoptic, Papanicolaou and H\&E, to the same pathologist only with the clinical diagnosis. After the final report of FNAB, the biopsy report was issued, serving as gold standard. After the calculations, the results of sensitivity, specificity and accuracy for Panoptic staining were $28.6 \%, 76 \%$ and $15.4 \%$, respectively. The result of sensitivity, specificity and accuracy for Papanicolaou staining were $71.4 \%, 76.7 \%$ and $23.3 \%$, respectively. The result of sensitivity, specificity and accuracy for H\&E staining were $82.1 \%, 23.3 \%, 28.6 \%$, respectively. We can conclude, according to the methodology of this study that, H\&E and Papanicolaou stains showed the same sensitivity of diagnosing malignant neoplasms. H\&E stain showed a better specificity for diagnosing benign neoplasms, compared with Papanicolaou and Panoptic stains. H\&E stain showed better accuracy, to give definitive diagnosis, followed by Papanicolaou and Panoptic stains.
\end{abstract}

Keywords: Biopsy, Fine-Needle; Sensitivity and Specificity; Staining and Labeling.

\section{Introduction}

Histological staining is a technique that facilitates microscopic examination of a tissue through differentiation of the color. The most commonly used stains in Fine Needle Aspiration Biopsy (FNAB) are the Romanowsky type stains (Panoptic), Papanicolaou and Hematoxylin-Eosin (H\&E), the latter being the least used in this technique. ${ }^{1}$

Hematoxylin is natural and has poor affinity in the tissue when used alone. It only become a dye when oxidized and its main product is haematein. ${ }^{2}$ Eosin is an acid dye from xanthene family, which stains all other tissues that Hematoxylin do not stains in a variety of bright pink, orange or red. ${ }^{2}$ Papanicolaou staining is polychromatic and shows variations in cell morphology and their degrees in cellular matrix and metabolic activity. ${ }^{2}$ 
The Romanowsky type stains are polychromatic and are based on the Romanowsky effect. These dyes are Wright, Giemsa, May-Grünwald-Giemsa and Diff-Quick $^{\circledR}$ (Wright-Giemsa or Panotic). ${ }^{2,3}$

The most commonly used stains in FNAB in oral cavity are the same of other regions, the Romanowsky type stains for air-dried smears and Papanicolaou and H\&E to slides fixed in alcohol. ${ }^{4,5,6,7}$

The study aims to evaluate the sensitivity, specificity and accuracy of FNAB in different staining techniques for nodular lesions from oral cavity and head and neck region.

\section{Methodology}

The Ethics Committee of FOUSP (Faculdade de Odontologia da Universidade de São Paulo) approved the research with the protocol 61/11 CAAE 0069.0.017.000-11. 46 consecutive patients who sought treatment at the Discipline of Clinical Stomatology of FOUSP with nodular lesions in the oral cavity and the head and neck region were selected.

Inclusion criteria were as follows: patients of both genders, all ethnicities, above 10 years old, without restriction of comorbidities and whom both FNAB and regular biopsy were performed. Exclusion criteria were patients under 10 years old and who only underwent FNAB without confirmation by regular biopsy.

After clinical examination and establishment of differential diagnosis, patients who had nodular lesions independent of size, color, consistency, presence of ulcerated surface or other clinical characteristics in the oral cavity and the head and neck region were prepared for FNAB and subsequently for incisional or excisional biopsy.

There was no need for the control group because it was a comparative method between the results from three staining techniques and the result of regular biopsy.

To perform FNAB a Franzen pistol type (Medpej ${ }^{\circledR}$, Ribeirão Preto, Brazil) was used, coupled to a 20 mL syringe $\left(\mathrm{BD}^{\circledR}\right.$, São Paulo, Brazil) and 23 or 25 gauge needles $\left(\mathrm{BD}^{\circledR}\right.$, São Paulo, Brazil; Terumo ${ }^{\circledR}$, São Paulo, Brazil).

The procedure was performed according to the technique described by Zadjicek. ${ }^{7,8}$ The area was primarily prepared aseptically and local anesthesia was used only if the biopsy was performed at the same time. The needle was inserted into the lesion, a vacuum was applied and the operator made back and forth movements with the needle to obtain a proper sample. The pressure was then released and the needle removed from the lesion. The syringe was withdrawn from the gun and the needle was removed from the syringe. Most of the material collected was in the needle and after removing the needle from the syringe, it was filled with air and the needle was placed near the surface of a glass slide, on which the material collected was deposited.

The material was deposited on 6 slides, then 2 slides were air dried and stained with the method of Panoptic and 4 slides were fixed in alcohol and sent to the Discipline of Oral Pathology of FOUSP with a differential diagnosis. The 4 fixed slides were stained with Papanicolaou and H\&E methods.

FNAB slides were evaluated by a pathologist without prior visualization of the histologic slides obtained by regular biopsy, but all FNAB slides were sent with a report of patient's data and clinical diagnosis of the lesion.

The results of the 6 FNAB slides were compared with the histologic slides in order to obtain results that demonstrated the sensitivity, specificity and accuracy of the method in different stains.

Sensitivity, specificity and accuracy was made in each stain group studied in FNAB, comparing their reports with their histologic reports.

\section{Results}

46 patients sought the Clinic of the Discipline of Clinical Stomatology at FOUSP but 39 patients examined and submitted to FNAB were evaluated for comparison of the three staining techniques. The other 7 patients were excluted because the material collected from FNAB was stained just by Papanicolaou and Hematoxilyn-Eosin. Of these 39 patients, 32 had benign lesions and 7 had malignant lesions.

Panoptic stain showed, 19 benign lesions, 2 malignant lesions and 18 inconclusive cases. Of those 18 inconclusive cases, 5 were malignant lesions, 
8 were benign lesions and 5 cases had insufficient material for the analysis. The result of FNAB in this staining was consistent with the result of the regular biopsy in 4 cases.

Papanicolaou stain showed 23 benign lesions, 5 malignant lesions and 11 inconclusive cases. Of those 11 inconclusive cases, 2 were malignant lesions, 7 were benign lesions and 2 cases had insufficient material for the analysis. The result of FNAB in this staining was consistent with the result of the regular biopsy in 7 cases.

H\&E stain showed 23 benign lesions, 5 malignant lesions and 11 inconclusive cases. Of those 11 inconclusive cases, 2 cases were malignant lesions, 4 cases were benign lesions and 5 cases had insufficient material for the analysis. The result of FNAB in this staining was consistent with the result of the regular biopsy in 6 cases.

There were no false-negative or false-positive results in any of the three staining techniques. For statistical analysis, inconclusive cases from malignant lesions were considered false-negative and inconclusive cases from benign lesions were considered false-positives. To determine accuracy, all cases with inconclusive report and no material for analysis were excluded from the sample.

Panoptic stain presented sensitivity of $28.6 \%$, specificity of $76 \%$ and accuracy of the $15.4 \%$. Papanicolaou stain presented sensitivity of $71.4 \%$, specificity of $76.7 \%$ and accuracy of $23.3 \%$. H\&E stain presented sensitivity of $71.4 \%$, specificity of $82.1 \%$ and accuracy of $28.6 \%$. These rates are shown in Tables 1 and 2.

Comparing therefore the three staining techniques, according to the methodology of this study, the ratios are:

- H\&E and Papanicolaou stains have the best sensitivity rate $(71.4 \%)$, compared to the Panoptic stain $(28.6 \%)$

- H\&E stain shows the best specificity (82.1\%) compared to the Papanicolaou stain $(76.7 \%)$ and Panoptic stain $(76 \%)$

- H\&E stain presents the best accuracy (28.6\%) compared with Papanicolaou stain (23.3\%) and Panoptic stain (15.4\%)
Table 1. Sensitivity and specificity for Papanicolaou, Panoptic and H\&E stains.

\begin{tabular}{lccc}
\hline Stains & Results & $\begin{array}{c}\text { Malignat } \\
\text { Regular Biopsy }\end{array}$ & $\begin{array}{c}\text { Bening Regular } \\
\text { Biopsy }\end{array}$ \\
\hline Papanicolaou & Malignat FNAB & 5 & 7 \\
Papanicolaou & Benign FNAB & 2 & 23 \\
Panoptic & Malignat FNAB & 2 & 8 \\
Panoptic & Benign FNAB & 5 & 19 \\
H\&E & Malignat FNAB & 5 & 4 \\
H\&E & Benign FNAB & 2 & 23 \\
\hline
\end{tabular}

Table 2. Comparision of the three stains techniques according to sensitivity, specificity and acuracy.

\begin{tabular}{lccc}
\hline Results & Panoptic & Papanicolaou & H\&E \\
\hline Sensitivity & $28,6 \%$ & $71,4 \%$ & $82,1 \%$ \\
Especificity & $76 \%$ & $76,7 \%$ & $23,3 \%$ \\
Acuracy* & $15,4 \%^{*}$ & $23,3 \%^{*}$ & $28,6 \%^{*}$ \\
\hline
\end{tabular}

*Inconclusive cases excluded from the sample

\section{Discussion}

In the present study, the staining techniques used were Panoptic, Papanicolaou and H\&E in order to compare which of them showed the best sensitivity, specificity and accuracy. In most studies, the three techniques, or two, one air dried and the other fixed by alcohol, are complementary for the diagnosis, without differentiating each stain technique. ${ }^{4,5,6,9,10,11,12}$

The procedure was performed according to the technique described by Zadjicek. ${ }^{78}$ The material was collected more than once when lesions were solid and only once, when a liquid content was present. Major bleeding or purulent contents were discarded in order to obtain a better visualization of the samples. All patients who fulfilled the inclusion criteria underwent the biopsy procedure, with subsequent histologic report in order to compare the FNAB report in the three techniques. All patients were referred for treatment after the issuance of the histologic report.

The length in the Panoptic technique is usually 1 minute according to manufacturer, and easy to perform and be visualized under a microscope..$^{13} \mathrm{In}$ the present study, there was no pathologist at time of collection, which hindered to view if the material collected was sufficient or not for analysis. This reflects 
in our rate of inconclusive and without sample to analyze, collected in each staining technique.

Many studies emphasize the presence of a pathologist at the time of collection or a cytotechnologist (capable of collecting and viewing the sample at the same time), demonstrating that these professionals are essential and accuracy of the technique reaches high levels, making it reliable for diagnostic, helping to decrease the time between diagnosis and treatment., ${ }^{7,1415}$

There were no false positive or false negative in our analysis as demonstrated by a few cases in the literature ${ }^{5,16}$ Despite being something positive from the analysis, it turned to be difficult to calculate sensitivity and specificity as reported, and did not reflect the truth in the study. Therefore, for purposes of statistical analysis, inconclusive cases from malignant neoplasms were considered false negatives and inconclusive cases from benign lesions were considered false positives.

Sensitivity, specificity and accuracy were made according to Trott definitions ${ }^{7,17}$ where sensitivity is the ability to identify malignant lesions and specificity is the ability of the test identifying benign lions. Benign lesions were considered benign neoplasms, non-neoplastic proliferative lesions, inflammatory processes and reactive lesions. The accuracy of the test was calculated as the amount of FNAB results similar to the results of regular biopsy.

Panoptic stain showed $28.6 \%$ of sensitivity, $76 \%$ of specificity and $15.4 \%$ of accuracy. Papanicolaou stain showed $71.4 \%$ of sensitivity, $76.7 \%$ of specificity and $23.3 \%$ of accuracy. H\&E stain, showed $71.4 \%$ of sensitivity, $82.1 \%$ of specificity and $28.6 \%$ of accuracy.

For these data we could infer, according to the methodology of this study, that H\&E and Papanicolaou stains had similar sensitivity for diagnosing malignant neoplasms, more than Panoptic stain. The specificity to identify benign lesions was higher in H\&E stain than in Papanicolau and Panotic stains and the accuracy was higher in H\&E stain, when compared with Papanicolaou and Panotic stains.

With this results we can conclute that FNAB with Papanicolaou and H\&E stains could be very important to stablish the prognosis and the diagnosis of suspected bening lesions in patients that presents health problems that contraindicate surgical procedure at that moment.
Anand et al. ${ }^{18}$ compared the stains used in intraoperative nodal imprint cytology for breast carcinoma. Giemsa, H\&E and Papanicoalou stains were used. In the study, the report is that these three concomitant staining techniques should be used to give the final diagnosis of these cases. The accuracy in Giemsa staining technique was $95.3 \%$, for H\&E staining technique was $90.6 \%$ and for Papanicolaou staining technique was $91.58 \%$. There was a higher rate of false positives and false negatives in the techniques of Giemsa and Papanicolaou staining than in H\&E technique. Giemsa showed better accuracy, sensitivity and specificity when compared to the others techniques. There is also a report that Giemsa and H\&E stains are most often used in this kind of evaluation. According to the author, Papanicolaou stain have a better demonstration of squamous differentiation and keratinization of the cells. The presence of dry artifacts is the most technical problem in slides fixed in alcohol. His conclusion is that the experience of the pathologist in a particular staining technique demonstrates in the results of the study. ${ }^{18}$

As in other studies, ${ }^{1,19}$ Panoptic stain, showed larger size cells and without background on the slides as an artifact of air-dried smears, facilitating cell display when the sample was sufficient for analysis. This technique was also good in demonstrating bacterial presence in the samples studied, as reported in other studies. ${ }^{19}$

Slides fixed in alcohol and stained with Papanicolaou and H\&E showed a greater preservation of cells, with better preservation of their morphology, like a histological section and H\&E stain, according to the pathologist who evaluated the slides, is the most that preserves these features.

Papanicolaou stain showed more artifacts than the others, being more difficult to see because of the color patterns that presents. In slides with cystic content or with hemorrhagic content, a granular appearance is seen, in shades of brown, making difficult to visualize the cells.

Slides of cystic contents, in all three stains, showed a quite typical amorphous clot, facilitating their diagnosis. Even though, in these cases, there was no cells present in the capsules and it was not possible to give a precise diagnosis of each cystic lesion. 
The epithelial cells are easily seen in Papanicolaou staining technique, because its structure colors in a green tone, very specific in this technique.

Ahmed et al. ${ }^{20}$ compared the utility of liquid-based cytology with direct smear for oral lesions, in Papanicolaou and May-Grünwald-Giemsa stains. Liquid based slides in those stains showed better quality when compared with direct smear slides, but slides stained with Papanicolaou showed even higher qualities. ${ }^{20}$

In our study, the slides stained with Panoptic and H\&E showed a superior quality of staining when compared with the slides stained by the Papanicolaou technique. All of this statesments are showned in the Figures 1, 2, 3, 4, 5 and 6 .

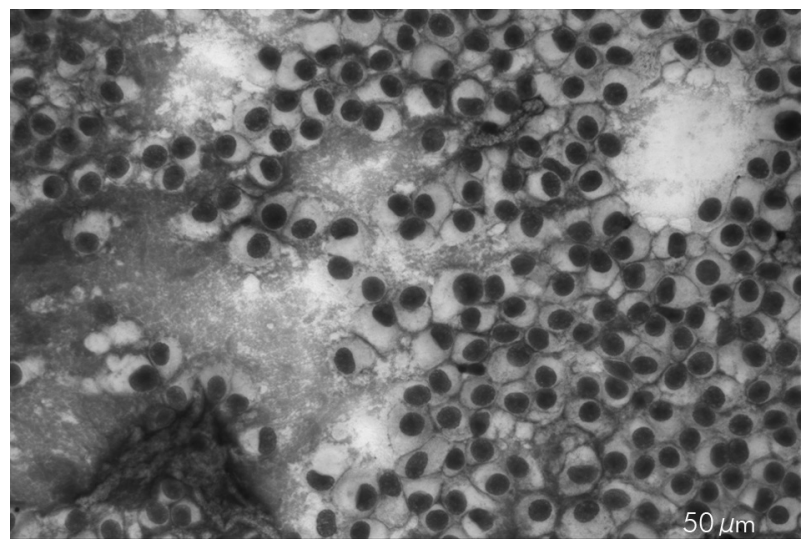

Figure 1. Presence of plasmocitoid cells on the smears with a sugestive report of Pleomorfic Adenoma (Panoptic stain).

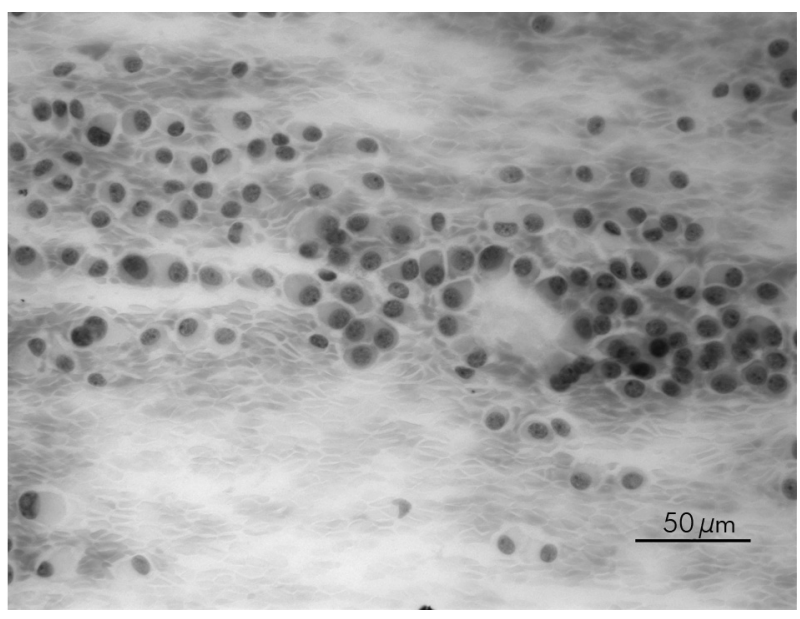

Figure 3. Presence of plasmocitoid cells on the smears with a sugestive report of Pleomorfic Adenoma (H\&E stain).
The FNA technique showed high accuracy in major and minor salivary gland lesions, to benign and malignant lesions as well as inflammatory lesions, in the three stains.

We must bear in mind that FNA is a simple and safe technique, presenting no risk to patients when performed or in the postoperative period. Expertise on the technique, combined with the presence of a pathologist at the time of collection, or even a trained clinician to review the material collected, significantly decrease the inconclusive sample, encouraging the use of Panoptic stain to a prior assessment of material collected.

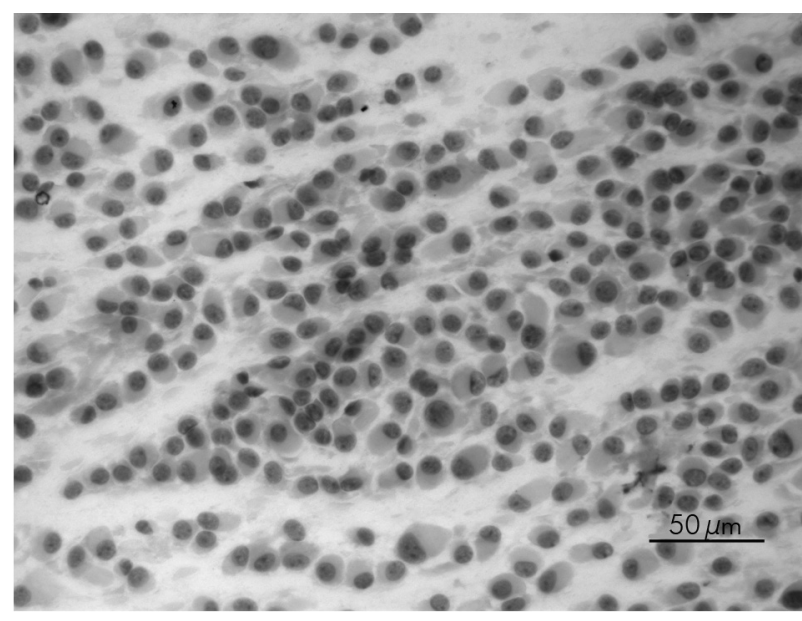

Figure 2. Presence of plasmocitoid cells on the smears with a sugestive report of Pleomorfic Adenoma (Papanicolaou stain).

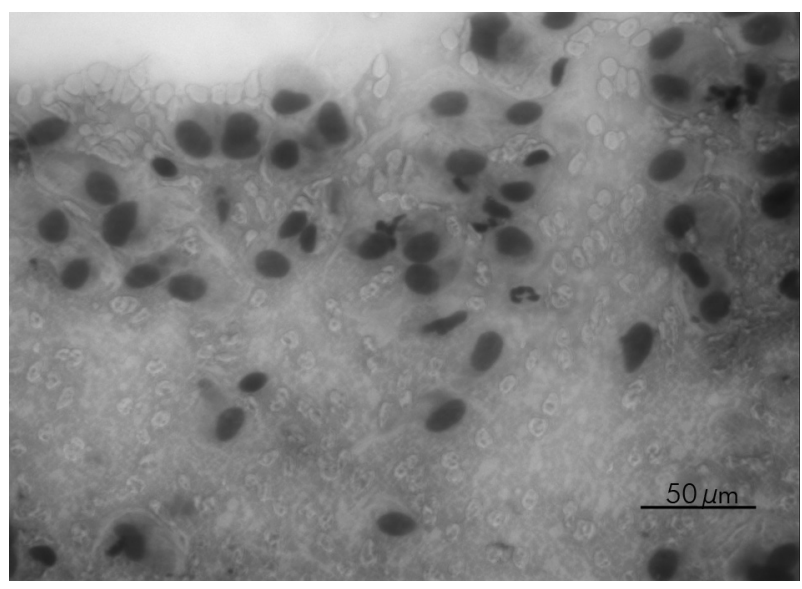

Figure 4. Presence of multinucleated atypical cells on the smears, with a sugestive report of mesenchimal malignat neoplasia (Panoptic stain). 


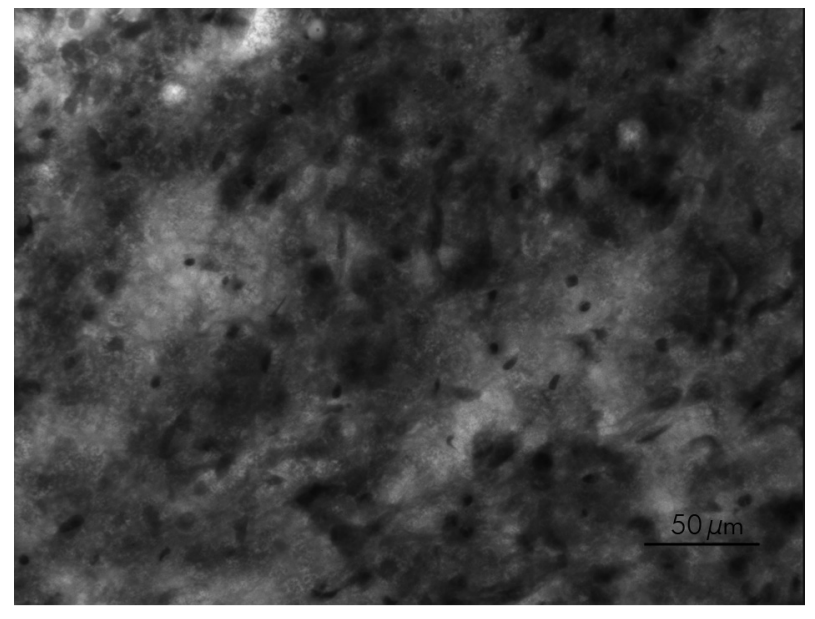

Figure 5. Presence of multinucleated atypical cells on the smears, with a sugestive report of mesenchimal malignat neoplasia (Papanicolaou stain).

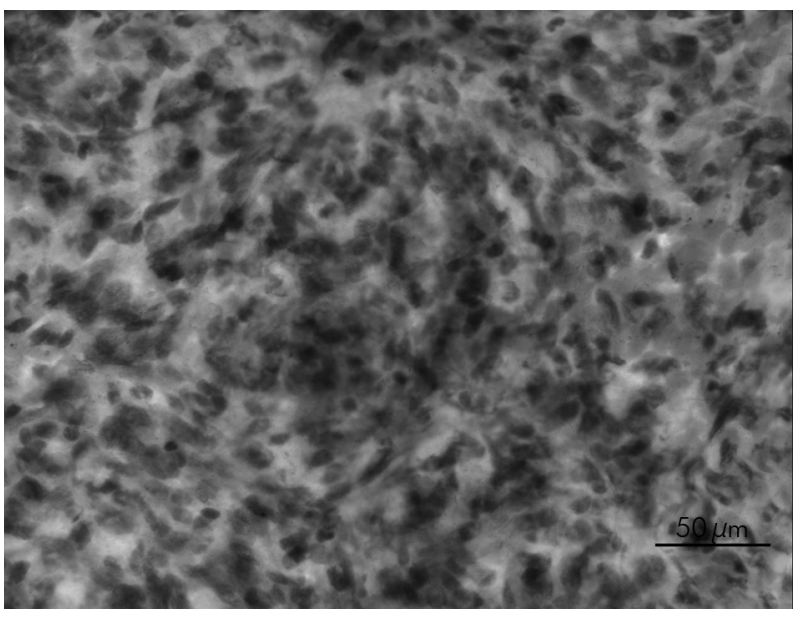

Figure 6. Presence of multinucleated atypical cells on the smears, with a sugestive report of mesenchimal malignat neoplasia (H\&E stain).

FNAB is a good preoperative tool, decreasing the time between the diagnosis of a lesion and its subsequent treatment, but we must bear in mind the indications and limitations that the technique offers.

Even when the use of FNAB is indicated, we must not fail to observe the clinical workup, collecting all medical and dental history as well as additional tests that we can use for the evaluation of a patient in particular. That helps us in a differential clinical diagnosis and to orientate the definitive diagnosis in FNAB.

In situations where a malignant neoplasm is suspected, in front of a benign or inconclusive diagnostic in FNAB, a biopsy should be performed, since it is a gold standard for the final and definitive diagnosis of lesions in general.

The experience of the pathologist to see slides of FNAB is essential for its diagnosis to be the most accurate, as well as familiarity with a particular technique of staining, helping thus considerably in the final diagnosis of the material collected by FNAB.

Even though it is not so commonly used in FNAB, H\&E stain should be considered by the pathologist at the time of viewing the slides, because it preserves similarities to histological cell structure.

We also encourage further studies with the use of ancillary techniques to refine the diagnosis, thus increasing the acuracy of the technique.

Even with all the limitations found in this study, the use of FNAB in the oral cavity and the head and neck region, under the legal limits of the dental practice should not be discouraged and new studies evaluating how this diagnostic tool is important, saved their proper indications, should be performed.

\section{Conclusion}

We can conclude, according to the methodology of this study, that:

- H\&E and Papanicolaou stains showed the same sensitivity for diagnosing malignancy.

- H\&E stain showed a better specificity for diagnosing benign neoplasms, compared with Papanicolaou and Panoptic stains.

- H\&E stain showed better accuracy, to give definitive diagnosis, followed by Papanicolaou and Panoptic stains. 


\section{References}

1. Ansari NA, Derias NW. Fine needle aspiration cytology. J Clin Pathol. 1997 Jul;50(7):541-3.

2. Jörundsson E, Lumsden JH, Jacobs RM. Rapid staining techniques in cytopathology: a review and comparison of modified protocols for hematoxylin and eosin, Papanicolaou and Romanowsky stains. Vet Clin Pathol. 1999;28(3):100-8. PubMed PMID: 12075519.

3. Bezrukov AV, editor. Romanowsky staining: on the question of priority [Internet]. Moscow: EMCO Ltd.; [cited 2015 May 18]. Available in: http://www.en.romanowsky.ru/site_files/ docs/K_voprosu_bezr_eng19e43.pdf

4. Castelli M, Gattuso P, Reyes C, Solans EP. Fine needle aspiration biopsy of intraoral and pharyngeal lesions. Acta Cytol. 1993 Jul-Aug;37(4):448-50.

5. Saleh HA, Clayman L, Masri H. Fine needle aspiration biopsy of intraoral and oropharyngeal mass lesions. Cytojournal. 2008 Mar 28;5:4. doi: 10.1186/1742-6413-5-4.

6. Fitzhugh VA, Maniar KP, Gurudutt VV, Rivera M, Chen H, $\mathrm{Wu} \mathrm{M}$. Fine-needle aspiration biopsy of granular cell tumor of the tongue: a technique for the aspiration of oral lesions. Diagn Cytopathol. 2009 Nov;37(11):839-42.

7. Santos AP, Sugaya NN, Pinto Junior DS, Lemos Junior CA. Fine needle aspiration biopsy in the oral cavity and head and neck region. Braz Oral Res. 2011 Mar-Apr;25(2):186-91.

8. Fulciniti F, Califano L, Zupi A, Vetrani A. Accuracy of fine needle aspiration biopsy in head and neck tumors. J Oral Maxillofac Surg. 1997 Oct;55(10):1094-7.

9. Schelkun PM, Grundy WG. Fine-needle aspiration biopsy of head and neck lesions. J Oral Maxillofac Surg. 1991 Mar;49(3):262-7.

10. Barnard NA, Paterson AW, Irvine GH, Mackenzie ED, White $\mathrm{H}$. Fine needle aspiration cytology in maxillofacial surgeryexperience in a district general hospital. Br J Oral Maxillofac Surg. 1993 Aug;31(4):223-6.

11. Fonseca FP, Ribeiro AC, Santos-Silva AR, Vargas PA, Lopes MA. Fine needle aspiration cytology and intralesional steroid injection in a central giant cell granuloma affecting the gingiva: a new clinical approach. Braz Dent J. 2013;24(4):420-7.
12. Cozzolino I, Vigliar E, Todaro P, Peluso AL, Picardi M, Sosa Fernandez LV, et al. Fine needle aspiration cytology of lymphoproliferative lesions of the oral cavity. Cytopathology. 2014 Aug;25(4):241-9. Epub 2014 Feb 7.

13. Platt JC, Davidson D, Nelson CL, Weisberger E. Fine-needle aspiration biopsy: an analysis of 89 head and neck cases. J Oral Maxillofac Surg. 1990 Jul;48(7):702-6; discussion 6-7.

14. Gomez-Macías GS, Garza-Guajardo R, Segura-Luna J, Barboza-Quintana O. Inadequate fine needle aspiration biopsy samples: pathologists versus other specialists. Cytojournal. 2009 Jun 18;6:9. doi: 10.4103/1742-6413.52831.

15. Reddy VM, Bennett WO, Bassett E, Cunliffe DJ, Fryer LC, Reece $\mathrm{PH}$, et al. On-site cytotechnician evaluation of the adequacy of fine needle aspiration in a neck lump clinic. Ann R Coll Surg Engl. 2013 Nov;95(8):595-8.

16. Robaina TC, Coutinho ACA, Valladares CP, Dias EP, Lourenço SQC. Correlationship of citopathology and histopathology on oral mucosa lesions after fine needle aspiration. Rev Col Bras Cir. 2007 Sep-Oct;34(5):285-9.

17. Florentine BD, Staymates B, Rabadi M, Barstis J, Black A, Hospital CCotHMNM. The reliability of fine-needle aspiration biopsy as the initial diagnostic procedure for palpable masses: a 4-year experience of 730 patients from a community hospital-based outpatient aspiration biopsy clinic. Cancer. 2006 Jul;107(2):406-16.

18. Anand M, Kumar R, Jain P, Asthana S, Deo SV, Shukla NK, et al. Comparison of three different staining techniques for intraoperative assessment of nodal metastasis in breast cancer. Diagn Cytopathol. 2004 Dec;31(6):423-6.

19. Krafts KP, Pambuccian SE. Romanowsky staining in cytopathology: history, advantages and limitations. Biotech Histochem. 2011 Apr;86(2):82-93.

20. Ahmed HG, Edris AM, Mohmed EA, Hussein MO. Value of centrifugated liquid-based cytology by Papanicolaou and May-Grünwald in oral epithelial cells. Rare Tumors. 2009 Jul 22;1(1):e12. 